Cuadernos de Documentación Multimedia

ISSN: 1575-9733

http://dx.doi.org/10.5209/CDMU.55626

\title{
Producción científica sobre cine indexada en la Web of Science en la categoría « Film, Radio \& Television » : 2000-2016
}

\author{
Rocío Gómez-Crisóstomoํㅜ ${ }^{1}$ Luz Mª Romo-Fernández ${ }^{2}$
}

Recibido: 26 de marzo de 2017 / Aceptado: 14 de abril de 2017

Resumen: El presente trabajo trata de analizar la producción científica contenida en la Web of Science en el periodo 2000-2016 sobre cine, publicada en fuentes indizadas bajo la categoría de Film, Radio, Television. Tras realizar las consultas pertinentes en la base de datos mencionada, se extraen un total de 12.514 trabajos, que son estudiados para analizar datos tales como número anual de documentos, países de afiliación de sus autores, categorías temáticas relacionadas, fuentes en las que se difunden los trabajos, idiomas de publicación, tipología documental y promedio de citas por documento.

Palabras clave: Cine; Web of Science; Producción científica.

[en] Scientific production about cinema indexed in the Web of Science in the category of “Film, Radio \& Television”: 2000-2016

\begin{abstract}
The present work tries to analyze the scientific production about cinema, contained in the Web of Science in the period 2000-2016, published in sources indexed under the category of Film, Radio, Television. After completing the relevant queries in the above mentioned database, a total of 12.514 works are extracted, which are studied to analyze data such as annual number of documents, countries of affiliation of their authors, related thematic categories, sources in which works are disseminated, languages of publication, type of documents and cites received by document.
\end{abstract}

Keywords: Cinema; Web of Science; Scientific production

Sumario: 1. Introducción 2. Objetivo 3. Metodología 4. Resultados 5. Conclusiones 6. Bibliografía

Cómo citar: Gómez-Crisóstomo, R.; Romo-Fernández, L.M. (2017). Producción científica sobre cine indexada en la Web of Science en la categoría “Film, Radio \& Televisión”: 2000-2016 Cuadernos de Documentación Multimedia, 28 (1), 62-72.

\footnotetext{
1 Departamento de Información y Documentación. Universidad de Extremadura (España)

E-mail: mrgomcri@unex.es

${ }^{2}$ Facultad de Ciencias de la Documentación y la Comunicación. Universidad de Extremadura (España)

E-mail: lmromfer@unex.es
} 


\section{Introducción}

Los intentos del hombre por captar la imagen en movimiento es consecuencia de haber logrado capturar la imagen fija, por lo que parece un paso lógico en la evolución de la captación de imagen. El primer antecedente para captar la imagen en movimiento es la utilización de la "cámara oscura", que se basaba en los inventos del siglo XIX tales como la fotografía y el daguerrotipo.

La historia del cine, entendida como espectáculo básico, comienza su andadura en la ciudad de París en el año 1895, donde los hermanos Luimière crearon el cinematógrafo partiendo del kinetoscopia inventado por Thomas Alva Edison (inventor además de la lámpara incandescente y el fonógrafo) (Sánchez, 2002). La evolución en estos tiempos ha sido espectacular, tanto en la forma de captación como de difusión, como la impresionante industria del entretenimiento que se ha generado a su alrededor. La evolución tecnológica facilita y agiliza los procesos de captación, distribución e incluso manipulación de los contenidos cinematográficos (Gubern, 1993).

Pero independientemente de factores industriales (Rimbau, 2011), económicos (Vogel, 2004) y culturales, el cine siempre ha sido una fábrica de sueños, una fórmula de mostrar la realidad -o aparentemente parecida- a unos asombrados espectadores, una forma de crear mundos alternativos, en definitiva, un método de expresión artística que denominamos "el séptimo arte".

Aunque el cine no deja de ser audiovisual, y nace con el fin de entretener, presenta un corpus científico derivado de lo complejo de una industria (Guback, 1980) con vertientes tan diversas como la tecnológica, la industrial, la empresarial, la artística, etc. e incluso la documentación (López, 1992) (Cuadra, 2013) (León, 2016).

Por ello, o derivado de dicha necesidad, existe un importante número de publicaciones científicas que se dedican a mostrar las diversas líneas de investigación, actuación y/o experiencias existentes en el ámbito cinematográfico.

Aunque inicialmente las revistas del ámbito cinematográfico suelen clasificarse dentro de las Artes y por lo tanto en las Humanidades, la transversalidad del cine hace posible que existan revistas que, desde otros prismas y otras áreas temáticas, se dedican de forma directa o indirecta a difundir los hitos científicos o experimentales de la cinematografía.

Por la importancia de la producción científica en la temática cinematográfica (junto con la radiofónica y la televisiva), la Web of Science ha creado una categoría donde aglutina los contenidos de las principales revistas de esta materia, siendo esta categoría la que se ha analizado con el fin de lograr los objetivos planteados en el trabajo. 


\section{Objetivo}

El objetivo principal de este estudio es analizar la producción científica contenida en la Web of Science en el periodo 2000-2016 sobre cine, publicada en fuentes indizadas bajo la categoría de Film, Radio, Television.

Para ello se tratará de:

- Conocer el número anual de documentos presentes en la Web of Science en el periodo estudiado.

- Identificar los países a los que pertenecen las instituciones de afiliación de los autores participantes en los trabajos analizados.

- Determinar las categorías temáticas de la WoS relacionadas con las publicaciones estudiadas.

- Extraer las fuentes elegidas por los autores para la difusión de sus trabajos.

- Conocer los idiomas en los que se publican los trabajos analizados.

- Establecer la tipología de los documentos objeto de estudio.

- Calcular el promedio de citas por documento recibidas por los trabajos analizados.

\section{Metodología}

Para llevar a cabo este estudio, en primer lugar, se procedió a realizar una serie de consultas en la colección principal de la base de datos Web of Science para identificar aquellas publicaciones pertenecientes a revistas de la categoría Film, Radio, Television, que tratasen sobre cine, búsqueda que fue limitada al periodo de tiempo 2000-2016. Para ello, se utilizaron los términos de búsqueda truncados cine* y film*. Se obtuvieron un total de 12.514 registros.

Si bien la categoría objeto de estudio no está presente en la actualidad en el Journal Citation Report, sí que aparece en la Web of Science, debido a que forma parte del Emerging Sources Citation Index (ESCI), un nuevo índice en la Colección de Web of Science Core, que amplía la cobertura de sus contenidos, con la finalidad de incluir las tendencias e innovaciones científicas todavía no identificadas como literatura científica de alto impacto. Proporciona visibilidad a todas las revistas revisadas por pares de importancia regional y en campos científicos emergentes que cumplen con unos criterios básicos de calidad para su indexación (revisión por pares, entre otros). Todas ellas están siendo evaluadas para su posible inclusión en las bases de datos con factor de impacto (Science Citation Index Expanded (SCIE), Social Science Citation Index (SSCI) y Arts and Humanities Citation Index (AHCI)).

Estos registros fueron incorporados a una base de datos diseñada ad-hoc, en la que posteriormente fueron realizadas las consultas necesarias para obtener resultados tales como el número de documentos por año y país, áreas de 
investigación, tipología documental e idioma de las publicaciones y fuentes en las que se publican los documentos analizados.

Los resultados de todas las consultas son plasmados en tablas y gráficas que facilitan su interpretación, y descritos y comentados para proporcionar un mejor entendimiento.

El rango de fechas entre el que se ha realizado la consulta ha sido entre el 21 al 23 de marzo de 2017.

\section{Resultados}

Se extraen un total de 12.514 documentos que tratan sobre cine y que están publicados en revistas de la categoría Film, Radio, Television de la Web of Science en el periodo 2000-2016. Como se observa en el gráfico 1, la producción estudiada muestra una tendencia estable a lo largo del periodo, siendo en los primeros y en los últimos años del periodo en los que más documentos se publican, superando tanto en el año 2002 como en el 2016 los mil documentos.



Gráfico 1. Evolución temporal de documentos sobre cine en WoS 
Tabla 1. Producción científica sobre cine en WoS por países ( $>2$ documentos)

\begin{tabular}{|c|c|c|c|}
\hline PAÍS & DOCUMENTOS & PAís & DOCUMENTOS \\
\hline USA & 2376 & Brazil & 16 \\
\hline England & 1162 & Norway & 14 \\
\hline Canada & 270 & Argentina & 10 \\
\hline Australia & 256 & Mexico & 9 \\
\hline Spain & 211 & Taiwan & 9 \\
\hline Scotland & 160 & Chile & 7 \\
\hline Belgium & 118 & Poland & 7 \\
\hline Germany & 102 & Portugal & 7 \\
\hline France & 90 & Nigeria & 6 \\
\hline Croatia & 73 & Pakistan & 6 \\
\hline Netherlands & 72 & Czech Republic & 5 \\
\hline Turkey & 69 & Romania & 5 \\
\hline Peoples R China & 60 & Trinid Tobago & 5 \\
\hline Sweden & 52 & Cyprus & 4 \\
\hline Wales & 46 & Greece & 4 \\
\hline New Zealand & 41 & Malaysia & 4 \\
\hline India & 40 & Philippines & 4 \\
\hline Italy & 39 & U Arab Emirates & 4 \\
\hline Denmark & 33 & Botswana & 3 \\
\hline Ireland & 33 & Costa Rica & 3 \\
\hline North Ireland & 27 & Hungary & 3 \\
\hline Finland & 24 & Kenya & 3 \\
\hline Israel & 24 & Luxembourg & 3 \\
\hline Switzerland & 23 & Serbia & 3 \\
\hline Singapore & 22 & Colombia & 2 \\
\hline Aus tria & 20 & Egypt & 2 \\
\hline South Korea & 20 & Malta & 2 \\
\hline Japan & 17 & Slovenia & 2 \\
\hline South Africa & 17 & & \\
\hline
\end{tabular}

La tabla 1 muestra los países en que están ubicadas las instituciones de afiliación de los autores que intervienen en los documentos analizados. Cabe destacar que, en 2.376 documentos (cerca del 20 \%) participa al menos un autor cuya institución de afiliación pertenece a Estados Unidos. Seguido de éste, y con una cifra muy inferior (1.162 documentos) se encuentra Inglaterra, con autores que participan en un 9,29 \% de los trabajos. A continuación, Canadá, Australia y España poseen autores cuya participación se sitúa en torno al 2 \%, quedando el resto de países por debajo de esta cifra. 


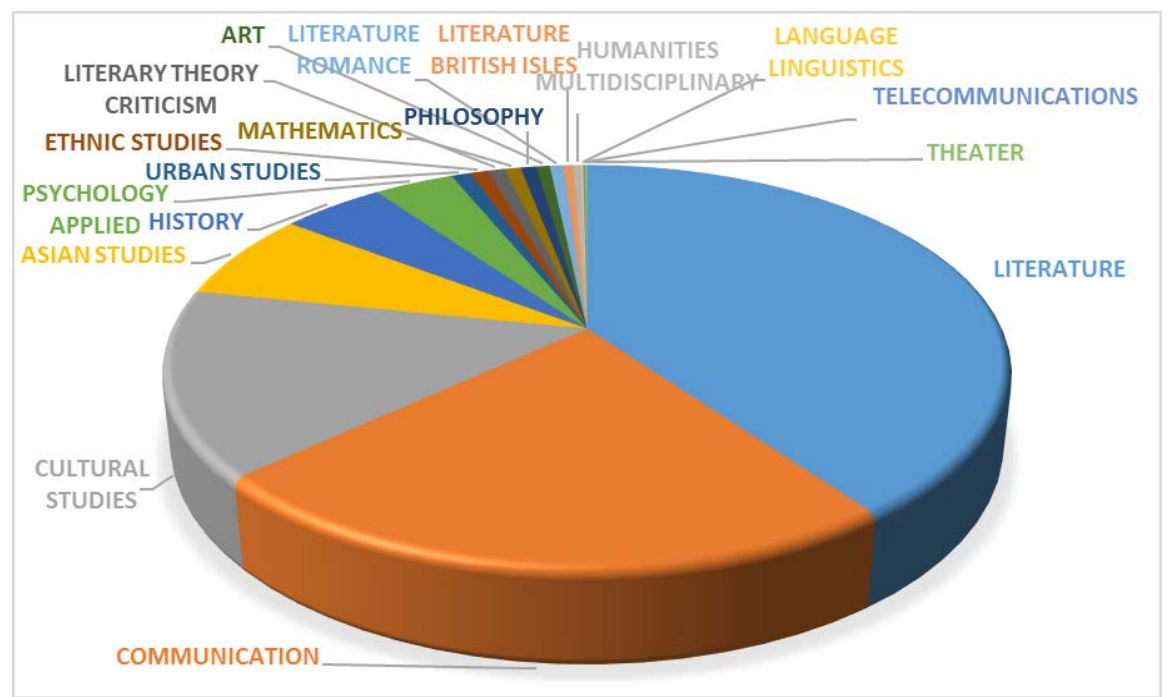

Gráfico 2. Producción científica sobre cine en WoS por categorías

Algunas de las fuentes categorizadas por la Web of Science dentro de Film, Radio, Television, además de estarlo en dicha categoría, también son encuadradas en alguna otra. En el caso objeto de estudio, de los 12.514 trabajos, 1.113 están publicados en fuentes pertenecientes a otra categoría. Estos datos vienen reflejados en el gráfico 2, en el que se aprecia que, de ellos, el $40 \%$ (445), están categorizados también dentro de Literature, cerca del $25 \%$ en Communication y el $15 \%$ en Cultural Studies. Les siguen Asian Studies, con un 7 \%, History, con un 4,76 \% y Pshychology Applied, con un 3,68 \%. El resto de categorías no alcanzan el $1 \%$ del total de trabajos categorizados en más de un área.

Tabla 2. Fuentes de publicación de la producción científica sobre cine en WoS (>10 documentos)

\begin{tabular}{|l|c|}
\hline \multicolumn{1}{|c|}{ FUENTE } & DOCUMENTOS \\
\hline Positif & 1901 \\
\hline Sight and Sound & 1494 \\
\hline Cineforum & 1357 \\
\hline Historical Journal of Film Radio and Television & 783 \\
\hline Film Comment & 717 \\
\hline Cineaste & 661 \\
\hline Film Quarterly & 660 \\
\hline
\end{tabular}




\begin{tabular}{|c|c|}
\hline FUENTE & DOCUMENTOS \\
\hline Screen & 511 \\
\hline Cinema Journal & 451 \\
\hline Hrvatski Filmski Ljetopis & 291 \\
\hline Journal of British Cinema and Television & 267 \\
\hline Journal of Popular Film and Television & 267 \\
\hline Kosmorama & 236 \\
\hline Film Criticism & 201 \\
\hline Literature Film Quarterly & 196 \\
\hline Atalante Revista de Estudios Cinematográficos & 190 \\
\hline Journal of Film and Video & 173 \\
\hline Continuum Journal of Media Cultural Studies & 147 \\
\hline Studies in French Cinema & 140 \\
\hline Adaptation the Journal of Literature on Screen Studies & 139 \\
\hline Animation an Interdisciplinary Journal & 116 \\
\hline Millennium Film Journal & 113 \\
\hline Canadian Journal of Film Studies Revue Canadienne D Etudes Cinematographiques & 110 \\
\hline Journal of Screenwriting & 107 \\
\hline Journal of Chinese Cinemas & 99 \\
\hline Moving Image & 84 \\
\hline Camera Obscura & 81 \\
\hline Bioscope South Asian Screen Studies & 79 \\
\hline Black Camera & 69 \\
\hline Historia y Comunicación Social & 52 \\
\hline Journal of Italian Cinema and Media Studies & 52 \\
\hline Cinemas D Amerique Latine & 48 \\
\hline Short Film Studies & 45 \\
\hline Film History & 44 \\
\hline Studies in Australasian Cinema & 42 \\
\hline Media Psychology & 41 \\
\hline Film International & 35 \\
\hline Journal of Scandinavian Cinema & 33 \\
\hline Journal of African Cinemas & 30 \\
\hline New Review of Film and Television Studies & 28 \\
\hline Projections the Journal for Movies and Mind & 27 \\
\hline Television New Media & 27 \\
\hline Studies in Spanish Latin American Cinemas & 26 \\
\hline Asian Cinema & 25 \\
\hline Film Philosophy & 24 \\
\hline Etudes Cinema tographiques & 23 \\
\hline Journal of Broadcasting Electronic Media & 22 \\
\hline Journal of African Media Studies & 20 \\
\hline New Soundtrack & 19 \\
\hline Music Sound and The Moving Image & 18 \\
\hline Metro & 17 \\
\hline Studies in Documentary Film & 17 \\
\hline Carmen from Silent Film to Mtv & 13 \\
\hline Critical Studies a Journal of Critical Theory Literature and Culture & 13 \\
\hline Con A de Animación & 11 \\
\hline Urban Cinematics Understanding Urban Phenomena Through the Moving Image & 10 \\
\hline
\end{tabular}


En la tabla 2 se observan las 56 fuentes en las que se publican 10 o más trabajos objeto de estudio. Sólo entre estas fuentes, suman un total de 12.402 publicaciones, es decir, más del 99 \% de las recogidas para este estudio. Como se aprecia, son las revistas Positif, de editorial francesa, Sight and Sound, inglesa, y Cineforum, italiana, las que aglutinan un mayor número de documentos. De estas 56, casi la mitad son de editorial inglesa, cerca del $25 \%$ americanas, y en torno al $5 \%$ francesas, españolas y escocesas.

Las revistas de editorial inglesa, recogen un total de 4.140 trabajos, es decir, el 33,38 \% de las publicaciones contempladas en la tabla; seguida de ésta, se encuentran las de editorial americana, con 3.620 documentos (29,19\%), francesa, con 1.972 e italiana, con 1.357 (15,90 \% y 10,94 \% respectiva mente).

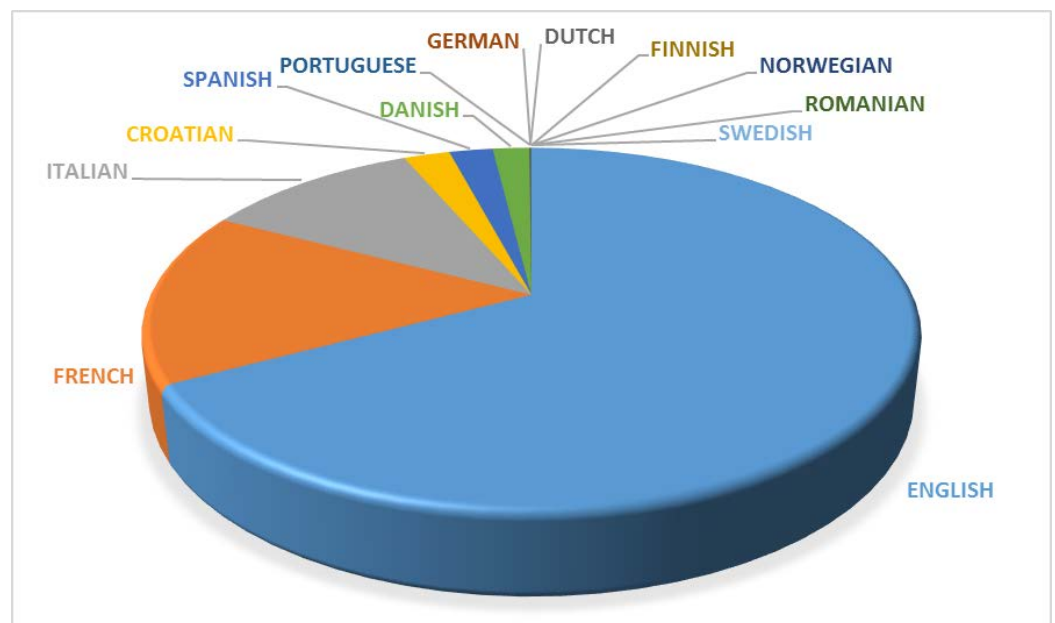

Gráfico 3. Idiomas de la producción científica sobre cine en WoS

El gráfico 3 aporta los diferentes idiomas en los que han sido publicados los trabajos. Era de esperar, a la vista de otros datos tales como afiliación de los autores y fuentes de procedencia, que estos datos dieran los siguientes resultados. Más del 65 \% de los trabajos son en inglés (lo que se traduce en 8.404 trabajos), lo que coincide con el patrón habitual de la actividad científica recogida en este tipo de base de datos. En segundo y tercer lugar se encuentran las publicaciones escritas en francés (con alrededor del $15 \%$ ) e italiano (con el 10,86 \% de los trabajos), datos que pueden relacionarse con los indicados en la tabla 1 y sus correspondientes comentarios, pues, como ya se dijo, a pesar de haber sólo 3 revistas de editorial francesa y una italiana, tanto una de las francesas (Postif), como la italiana (Cineforum), además de tener una larga trayectoria que cubre todo el periodo objeto de estudio, también cuentan con una periodicidad mensual, lo que hace que el número de trabajos en ellas publicados sea tan elevado.

A pesar de haber tres fuentes de nacionalidad española, ninguna de ellas aglutina un elevado número de documentos, debido a que tienen una periodicidad anual o semestral, y que, además, no todas ellas cubren el periodo 
de estudio completo, por lo que el porcentaje que suponen los documentos en este idioma no alcanza el 2,5\%.

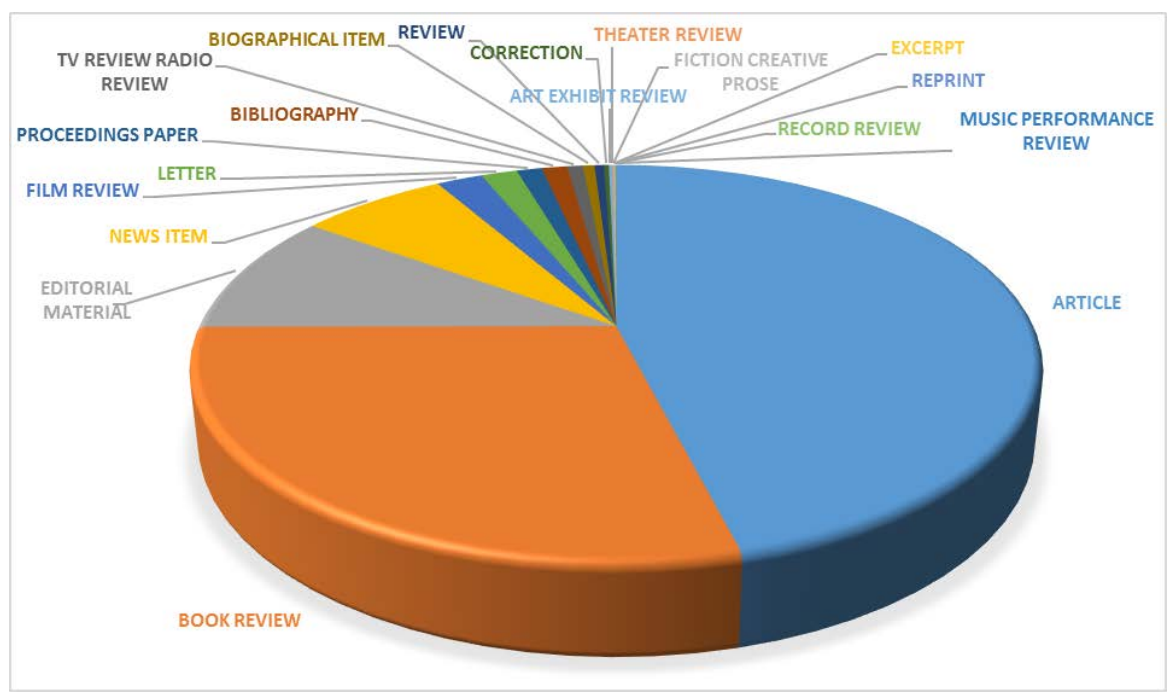

Gráfico 4. Tipo de documento de la producción científica sobre cine en WoS

El gráfico 4 muestra los tipos de documentos elegidos por los autores para publicar sus trabajos en la categoría y temática objeto de estudio. Como se observa, el artículo es el tipo documental predominante, con un 46 \%, patrón habitual en la mayor parte de las categorías científicas. No obstante, destaca el hecho de que las book reviews o reseñas bibliográficas obtengan un porcentaje tan alto (cerca del 30 \%). Esto está provocado por la manera de comunicación habitual en esta disciplina. El libro, no recogido claramente en la WoS, es una de las formas preferidas por los autores para presentar sus trabajos, en muchos casos más reflexivos que investigadores. De ahí que se produzca una gran cantidad de material en formato libro y que sea muy valorado por parte de la comunidad científica. Por ello, las revistas científicas incluyen un importante número de revisiones y reseñas bibliográficas a título informativo y de difusión de información, con el fin de dar a conocer las últimas publicaciones en formato libro en esta disciplina. 


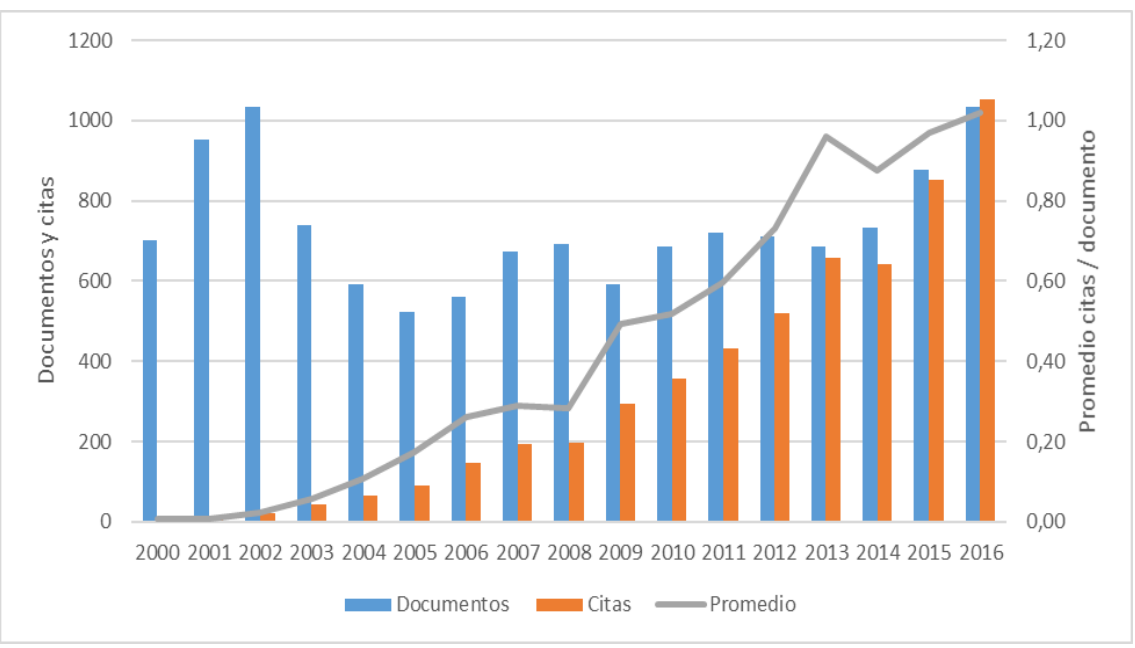

Gráfico 5. Citas por documento

En el gráfico 5 se comparan el número total de documentos con el número total de citas por ellos recibidas, así como el promedio anual de citas por documento. Cabe destacar los bajos valores obtenidos por los promedios de citas por documento, que sólo en el año 2016 alcanzan la cifra de 1 cita por documento. Este hecho puede relacionarse con la gran cantidad de documentos de tipo book review o reseña bibliográfica, tipo documental poco susceptible de recibir citas. Se muestra de este modo, la poca tendencia a la citación entre autores de la categoría estudiada, pues los promedios habituales de otras categorías suelen ser superiores.

\section{Conclusiones}

El número de documentos sobre cine, recogidos en las revistas de la categoría Film, Radio, Television de la base de datos Web of Science, y publicados entre los años 2000-2016, muestra una tendencia estable a lo largo de dicho periodo, con cifras sólo destacables en los años 2001, 2002 y 2016.

La mayor parte de los trabajos cuentan con al menos un autor afiliado a una institución americana o inglesa, con cifras cercanas al $20 \%$ y $10 \%$ respectivamente.

De los trabajos estudiados y pertenecientes a revistas de la categoría Film, Radio, Television, sólo el 8,89 \% están además encuadrados en alguna otra categoría temática de WoS, principalmente en Literature, Communication y Cultural Studies.

Las fuentes que recogen un mayor número de documentos son Positif, de editorial francesa, Sight and Sound, inglesa, y Cineforum, italiana. Casi la mitad de las fuentes elegidas por los autores para sus publicaciones son de editorial inglesa, seguidas de las americanas.

$\mathrm{Al}$ igual que ocurre en la mayor parte de las disciplinas, más del $65 \%$ de los trabajos publicados están en inglés. En segundo y tercer lugar se encuentran las 
publicaciones escritas en francés (con alrededor del $15 \%$ ) e italiano (con el 10,86 $\%$ de los trabajos).

Los tipos de documentos elegidos por los autores para publicar sus trabajos en la categoría y temática objeto de estudio son, el artículo, con un $46 \%$, seguida de las book reviews o reseñas bibliográficas, con cerca del $30 \%$.

El promedio de citas por documento presenta unos valores bastante bajos, alcanzando sólo en 2016 la cifra de 1 cita por documento.

\section{Bibliografía}

Cuadra, E. (2013). Documentación cinematográfica: roles y fuentes del documentalista en el cine. Barcelona: UOC.

León-Moreno, J.A.; Zapico-Alonso, F.; Caldera-Serrano, J. (2016). ¿Archivos audiovisuales aún sin digitalizar? Seguridad, preservación y amortización. Cuadernos de Documentación Multimedia, 27 (2), pp. 178-190.

López Yepes, A. (1992). Manual de documentación audiovisual. Pamplona: Ediciones Universidad de Navarra.

Guback, T.H. (1980). La industria internacional del cine. Madrid: Fundamentos.

Gubern, R. (1993). Historia del cine. Barcelona: Lumen, 1993.

Riambau, E. (2011). Hollywood en la era digital: de Jurassic Park a Avatar. Madrid: Cátedra, 2011.

Sánchez Noriega, J.L. (2002). Historia del cine: teoría y géneros cinematográficos, fotografía y televisión. Madrid: Alianza.

Vogel, H.L. (2004). La industria de la cultura y el ocio: un análisis económico. Madrid: Fundación Autor. 